\title{
MONOTONE RETRACTS AND SOME CHARACTERIZATIONS OF DENDRITES
}

\author{
G. R. GORDH, JR. AND LEWIS LUM
}

\begin{abstract}
Let $M$ be a metric continuum containing a fixed point $p$. The following conditions are shown to be equivalent. (i) $M$ is a dendrite. (ii) Each subcontinuum of $M$ is a monotone retract of $M$. (iii) $M$ is arcwise connected and each subcontinuum of $M$ containing $p$ is a monotone retract of $M$.
\end{abstract}

1. Introduction. The second author has proved that each of the following conditions is both necessary and sufficient for a dendroid $M$ to be a dendrite [3, Theorem 2.3], [4, Theorem 3].

(a) Each subcontinuum of $M$ is a monotone retract of $M$.

(b) Each subcontinuum of $M$ which contains a fixed point $p$ is a monotone retract of $M$.

The main purpose of this note is to prove that condition (a) characterizes dendrites among arbitrary metric continua, and that condition (b) characterizes dendrites among arcwise connected metric continua.

All of the results are established in the setting of Hausdorff continua.

2. Definitions. A continuum is a compact connected Hausdorff space. The reader is referred to [2] for basic properties of continua and undefined terms.

An $\operatorname{arc}[p, q]$ (sometimes generalized arc or ordered continuum) is a continuum which is irreducibly connected between the points $p$ and $q$. The continuum $M$ is said to be hereditarily unicoherent at $p$ if for each pair of subcontinua $H$ and $K$ containing $p, H \cap K$ is connected. An arboroid (dendroid) is a hereditarily unicoherent, arcwise connected (metric) continuum. A tree (dendrite) is a hereditarily unicoherent, locally connected (metric) continuum. A subset $Z$ of a topological space $X$ is a monotone retract of $X$ if there exists a mapping $r: X \rightarrow Z$ which is both monotone and a retraction.

\section{Some characterizations of trees.}

LEMMA. Let $M$ be a continuum containing a fixed point $p$. If each subcontinuum of $M$ containing $p$ is a monotone retract of $M$, then $M$ is hereditarily unicoherent at $p$ and connected im kleinen at $p$.

Proof. We first show that $M$ is unicoherent. If not, there exist subcontinua

Presented to the Society, January 22, 1976; received by the editors July 21, 1975 and, in revised form. October 6, 1975.

AMS (MOS) subject classifications (1970). Primary 54F50; Secondary 54C15.

Key words and phrases. Continuum, dendrite, tree, monotone retract, hereditary unicoherence at $p$. 
$H$ and $K$ such that $M=H \cup K$ and $H \cap K$ is not connected. Assume that $p \in H$, and let $r: M \rightarrow H$ be a monotone retraction. Choose $y \in r(K)-K$. Then $r^{-1}(y)$ is a subcontinuum which meets $H-K$ and $K-H$. Consequently, $r^{-1}(y)$ meets $H \cap K$, contradicting the fact that $r$ fixes $H \cap K$.

Since monotone maps clearly preserve unicoherence (use the fact that inverse images of connected sets are connected), $M$ is hereditarily unicoherent at $p$.

Suppose that $M$ is not connected im kleinen at $p$, and let $U$ be an open neighborhood of $p$ which contains no connected neighborhood of $p$. Let $C$ denote the component of $\operatorname{cl}(U)$ containing $p$, and let $r: M \rightarrow C$ be a monotone retraction. Choose an open set $V \subseteq U$ such that $p \in V$ and $r(V) \subseteq U$. Choose $x \in V-C$. Applying Theorem 2-16 of [2], it is easy to see that

$$
\left(r^{-1}(r(x)) \cap C\right) \cap \text { bd }(U) \neq \varnothing .
$$

But this is a contradiction since $r^{-1}(r(x)) \cap C=\{r(x)\} \subseteq U$.

TheOREM. Let $M$ be a continuum containing a fixed point $p$. The following conditions are equivalent.

(i) $M$ is a tree.

(ii) Each subcontinuum of $M$ is a monotone retract of $M$.

(iii) $M$ is arcwise connected and each subcontinuum of $M$ containing $p$ is a monotone retract of $M$.

(iv) $M$ is an arboroid and each arc $[p, q]$ in $M$ is a monotone retract of $M$.

Proof. That (i) implies each of the other conditions follows from [3, Theorem 2.1, p. 332].

(ii) implies (i). According to the Lemma, $M$ is hereditarily unicoherent at each point and connected im kleinen at each point. Thus $M$ is hereditarily unicoherent and locally connected [2, Theorem 3-11, p. 114], i.e., $M$ is a tree.

(iii) implies (iv). By the Lemma, $M$ is hereditarily unicoherent at $p$. Since $M$ is arcwise connected, $M$ is hereditarily unicoherent [1, Theorem 2.2], i.e., $M$ is an arboroid.

(iv) implies (i). See [4, Theorem 2.3].

REMARK. The assumption that $M$ is arcwise connected in (iii) of the Theorem can be replaced by any property of trees which implies arcwise connectivity in the presence of hereditary unicoherence at a point. Two such conditions are semiaposyndesis [1, Theorem 2.3] and continuum chainability [5, Theorem 1]. However, some such condition is necessary, for if $M$ denotes the " $\sin (1 / x)$ curve" (i.e., $M$ is the closure in the plane of the set $\{(x, y) \mid y=\sin (1 / x) ; 0<x \leqslant 1\})$, then $M$ contains a point $p$ such that each subcontinuum of $M$ containing $p$ is a monotone retract of $M$.

\section{REFERENCES}

1. G. R. Gordh, Jr., Concerning closed quasi-orders on hereditarily unicoherent continua, Fund. Math. 78 (1973), no. 1, 61-73. MR 48 \#1196. 
2. J. G. Hocking and G. S. Young, Topology, Addison-Wesley, Reading, Mass., 1961. MR 23 \# A2857.

3. Lewis Lum, A characterization of local connectivity in dendroids, Studies in Topology, Academic Press, New York, 1975, pp. 331-338.

4. - Order preserving and monotone retracts of a dendroid (submitted).

5. L. E. Rogers, Arcwise connectedness and continuum chainability (submitted).

Department of Mathematics, Guilford College, Greensboro, North Carolina 27410

Department of Mathematics, Salem College, Winston-Salem, North Carolina 27108 